\title{
A thermally cured 9,9-diarylfluorene-based triaryldiamine polymer displaying high hole mobility and remarkable ambient stability
}

\author{
Chi-Yen Lin, ${ }^{a}$ Yu-Cheng Lin, ${ }^{b}$ Wen-Yi Hung, ${ }^{* b}$ Ken-Tsung Wong, ${ }^{* a}$ Raymond C. Kwong, ${ }^{c}$ Sean C. Xia, ${ }^{c}$ \\ Yu-Hung Chen ${ }^{d}$ and Chih-I Wu ${ }^{d}$
}

\author{
Received 16th January 2009, Accepted 27th March 2009 \\ First published as an Advance Article on the web 21st April 2009 \\ DOI: $10.1039 / b 900977 a$
}

\begin{abstract}
We have synthesized and characterized a novel thermally polymerizable triaryldiamine monomer (VB-FNPD) possessing a styrene-functionalized 9,9-diarylfluorene core and have used time-of-flight transient photocurrent techniques to investigate the hole transport properties of its solution-processed and subsequently thermally cured $\left(170^{\circ} \mathrm{C}\right)$ polymer films. This novel polymeric material exhibits nondispersive hole transport behavior with a high hole drift mobility (up to $10^{-4} \mathrm{~cm}^{2} \mathrm{~V}^{-1} \mathrm{~s}^{-1}$ ). The film displayed remarkable ambient stability, even when exposed to air for one month. We tested the thermally generated polymer film as a hole transport material in organic light-emitting diodes incorporating tris(8-hydroxyquinolate) aluminium $\left(\mathrm{Alq}_{3}\right)$ as the emission and electron transport layer. The device exhibited a maximum external quantum efficiency $\left(\eta_{\mathrm{ex}}\right)$ of $1.4 \%$, significantly better than that of the device prepared using the corresponding model compound VB-model $\left(\eta_{\mathrm{ex}}=1.1 \%\right)$.
\end{abstract}

\section{Introduction}

Most organic light-emitting diodes (OLEDs) are configured as multilayer structures $^{1}$ in which each layer addresses a specific function, such as charge injection, transport, and emission. Historically, it has been difficult to prepare polymer-based OLEDs in multilayer structures because solution processing has typically been used to form films; i.e., the deposition of a new polymer film can lead to mixing and/or corroding of the previously deposited layers. ${ }^{2}$ Much effort has been exerted to overcome this limitation. For example, one promising approach toward forming multiple layers through solution processing is to employ alternating solvents of orthogonal polarities; ${ }^{3}$ e.g., in the deposition of conjugated polyelectrolytes collocated with neutral conjugated polymers. Another plausible approach is the use of an organic-soluble precursor that becomes insoluble upon selfassembly $^{4}$ or photochemical, ${ }^{5}$ electrochemical, ${ }^{6}$ or thermal ${ }^{7,8}$ treatment; many materials possessing various functional moieties - such as siloxane, oxetane, styrene, and trifluorovinyl ether units - have been developed as good candidates. ${ }^{9}$

Recently, Jen et al. reported that styryl groups are promising thermal curable moieties for the preparation of cross-linkable hole transport materials (HTMs). ${ }^{8}$ The precursor possessing the styryl moiety has the advantageous property that it can undergo mild thermal polymerization in the absence of an initiator. In addition, the resulting polymer networks exhibit high solvent resistance, making them suitable materials for the deposition of subsequent layers through solution processing. Despite the

a Department of Chemistry, National Taiwan University, Taipei 106, Taiwan. E-mail: kenwong@ntu.edu.tw; Fax: +886 2 33661667; Tel: +886233661665

${ }^{b}$ Institute of Optoelectronic Sciences, National Taiwan Ocean University, Keelung 202, Taiwan.E-mail: wenhung@mail.ntou.edu.tw

'Universal Display Corporation, 375 Phillips Blvd, Ewing, NJ 08618, USA

${ }^{d}$ Department of Electrical Engineering and Graduate Institute of Electrooptical Engineering, National Taiwan University, Taipei 106, Taiwan successful application of styrene-based HTMs, their intrinsic charge transporting characteristics and stability remain poorly understood. Indeed, the relationship between their molecular structures and charge carrier mobilities remains to be investigated..$^{10,11}$ In this paper, we report the synthesis, characterization, and detailed hole mobility studies of a novel 2,7-disubstituted fluorene-based triaryldiamine (VB-FNPD) bearing two pendent thermally polymerizable styryl groups. The polymerized film exhibits not only remarkable hole transport properties $\left(\mu_{\mathrm{h}}=c a\right.$. $10^{-4} \mathrm{~cm}^{2} \mathrm{~V}^{-1} \mathrm{~s}^{-1}$ ) but also high stability, even after storage for one month under an ambient environment. Parallel device comparisons employing a corresponding model compound revealed the potential of using VB-FNPD as a promising hole transport material in OLEDs.

\section{Results and discussion}

\section{Synthesis}

Scheme 1 depicts the synthesis of the monomer VB-FNPD, which is a derivative of the well-established hole-transport material $N, N^{\prime}$-di(1-naphthyl)- $N, N^{\prime}$-diphenylbenzidine ( $\alpha$-NPD). The diarylamino groups were attached to the coplanar rigid fluorene core to modulate the electronic properties and enhance the thermal stability. ${ }^{12}$ More importantly, we introduced two polymerizable vinylbenzyl (styryl) ether groups onto the peripheral phenyl substituents of the central fluorene unit, so as not to perturb the structure on the active main chromophore. Our synthesis began with the amination of the dibromide $\mathbf{1}^{13}$ with $N$-phenyl-1-naphthylamine in the presence of a catalytic amount of $\mathrm{Pd}(\mathrm{OAc})_{2}$ and tri-tert-butylphosphine to afford the bisphenol $\mathbf{2}$ in $82 \%$ yield. Subsequent etherification of $\mathbf{2}$ with 4 -vinylbenzyl chloride under basic conditions gave VB-FNPD as a yellow solid in $54 \%$ yield. We employed VB-model ${ }^{12}$ as a model compound for VB-FNPD to compare its physical properties and device characteristics. 

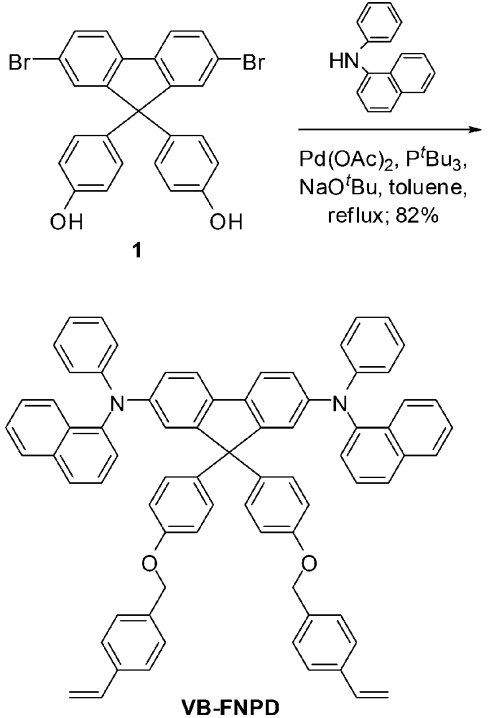

Scheme 1 Synthetic route toward VB-FNPD, and the structure of VB-model.

\section{Physical properties}

We used differential scanning calorimetry (DSC) to determine the thermal properties of VB-FNPD. As indicated in Fig. 1, the first scan of pristine VB-FNPD exhibited a glass transition temperature $\left(T_{\mathrm{g}}\right)$ of $c a .113^{\circ} \mathrm{C}$, lower than that of VB-model (129 $\left.{ }^{\circ} \mathrm{C}\right) .{ }^{12}$ In addition, the broad exothermic peak at $c a .166{ }^{\circ} \mathrm{C}$ corresponds to the thermal polymerization of VB-FNPD. In contrast, the second scan features no apparent peaks that can be detected at temperatures up to $250{ }^{\circ} \mathrm{C}$, implying the existence of a high degree of cross-linking between the styryl groups.

Fig. 2 displays UV-Vis absorption and photoluminescence spectra of the vacuum-deposited VB-model and dip-coated VBFNPD thin films before and after thermal treatment. The absorption spectra of the VB-FNPD films [both as-deposited (solution-processed) and thermally polymerized] matched quite well with that of VB-model. This finding reveals that the $\mathrm{sp}^{3}$ hybridized C9 carbon atom of the fluorene core impeded any $\pi$ orbital interactions between the chromophore and the pendent aryl groups; thus, the electronic transitions were not affected after introducing the two styrene moieties. In the emission

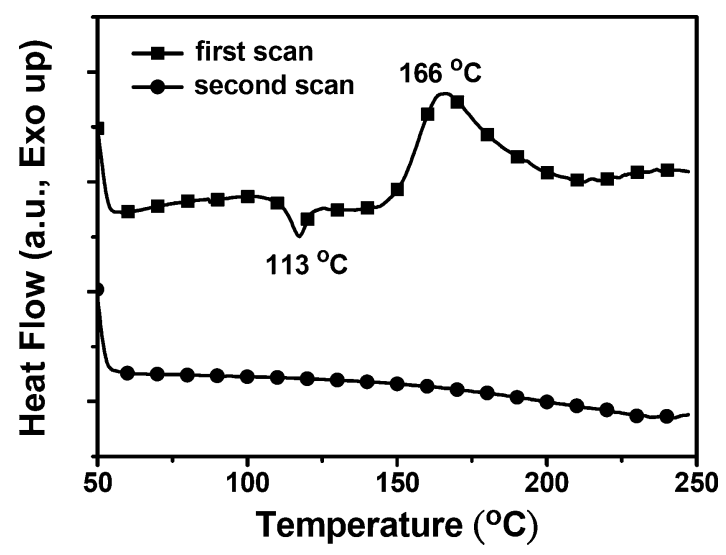

Fig. 1 DSC analysis of VB-FNPD.

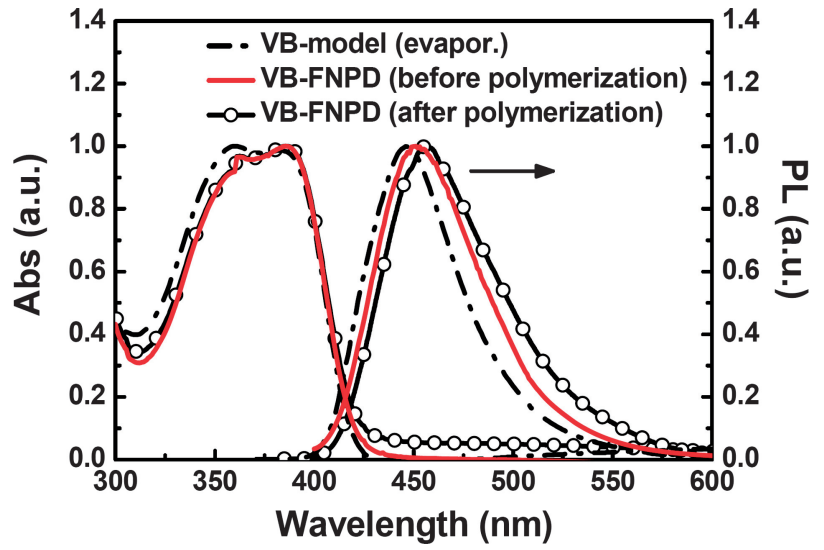

Fig. 2 UV-Vis absorption and photoluminescence spectra of thin films of VB-FNPD and vacuum-deposited VB-model.

spectra, however, the polymerized VB-FNPD thin film exhibited a slightly red-shifted maximum signal intensity (by $c a .10 \mathrm{~nm}$ ) and broader half-widths of the emission peak relative to that of VB-model. We attribute these differences to changes in the dielectric environment after thermal treatment; i.e., because chromophores surrounded by polystyrene chains should behave slightly different from those of model compounds in their respective excited states. Nevertheless, we cannot exclude the possibility of weak interchromophore interactions leading to the long-wavelength emission from the polymer films after hightemperature thermal polymerization.

\section{Electrochemical characterization}

We employed cyclic voltammetry (CV) to assess the electrochemical properties of VB-FNPD and VB-model (Fig. 3). The oxidation CV traces of the VB-model and VB-FNPD monomers in solution were almost the same, with each displaying two reversible oxidation potentials at 0.64 and $1.00 \mathrm{~V}$. The significant potential difference between the first and second oxidations 


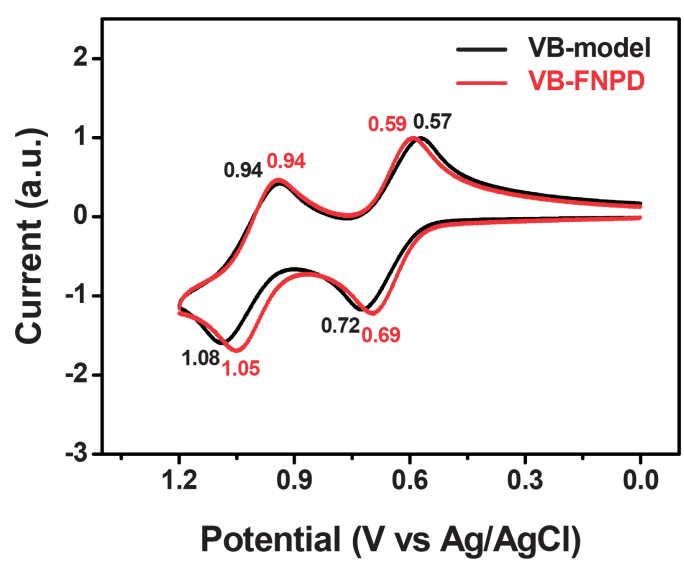

Fig. 3 Cyclic voltammograms of VB-model and VB-FNPD in solution.

clearly indicates that the removal of electrons occurred from the same chromophore. In addition, UV photoelectron spectroscopy (UPS) studies of thin films of VB-model and the non-polymerized and polymerized VB-FNPD revealed no differences in the energy levels of their respective highest occupied molecular orbitals (HOMOs; each $c a .-5.3 \mathrm{eV}$ ). Taken together, these results support the notion that the introduction of pendent polymerizable groups as peripheral substituents on the fluorene core did not alter the electrochemical properties of the active chromophore to any significant extent.

\section{Hole mobility measurements}

We used time-of-flight (TOF) transient photocurrent techniques to investigate the hole-transport properties of VB-model and the thermally polymerized VB-FNPD. The TOF transient of VBmodel for holes (Fig. 4a) displays a clear plateau, which indicates non-dispersive transport behavior; exposure of the VB-model film to an ambient environment led, however, to highly dispersive hole transport. We suspect that the penetrated oxygen and $\mathrm{H}_{2} \mathrm{O}$ acted as an extrinsic dopant and induced carrier trapping. We observed a similar non-dispersive photocurrent transient for the polymerized VB-FNPD [Fig. 4(b)]; under ambient conditions (exposure of the sample to air for 1 month), however, the nondispersive photocurrent transient was barely affected. After exposing the sample to sunlight for 1 or 5 days, we found that the TOF photocurrents degraded significantly, but the hole mobilities remained unaffected (i.e., they possess the same transit time $t_{\mathrm{T}}$ ). The dispersion of a sheet of carriers migrating through the sample can be obtained from the tail-broadening parameter, $w=$ $\left(t_{1 / 2}-t_{\mathrm{T}}\right) / t_{1 / 2}$, where $t_{\mathrm{T}}$ is the carrier transit time and $t_{1 / 2}$ is the time when the current has decayed to half of the plateau value. ${ }^{14}$ From Fig. 4(b), we obtain almost the same $w$ of 0.37 , from a transit time $t_{\mathrm{T}}=3.2 \mu \mathrm{s}$ and decay time $t_{1 / 2}=5.1 \mu \mathrm{s}$. These observations indicate that irradiation with sunlight only impeded the generation of charge carriers nearby the transparent electrode; it had a less pronounced effect on the carrier's drift properties. Thus, it is clear that thermal treatment of VB-FNPD generated a polymer that could effectively suppress the penetration of oxygen and water. The excellent air stability of the thermally polymerized VB-FNPD suggested that it has potential for application in organic optoelectronic devices.

Fig. 5 displays the hole mobilities of the vacuum-deposited VB-model and the dip-coated and thermally cured VB-FNPD polymer films plotted as functions of the square root of the electric field. VB-model exhibited hole mobilities from $5 \times 10^{-4}$ to $1 \times 10^{-3} \mathrm{~cm}^{2} \mathrm{~V}^{-1} \mathrm{~s}^{-1}$ at electric fields between $1.2 \times 10^{5}$ and $4 \times 10^{5} \mathrm{~V} \mathrm{~cm}^{-1}$, and from $5 \times 10^{-5}$ to $4 \times 10^{-4} \mathrm{~cm}^{2} \mathrm{~V}^{-1} \mathrm{~s}^{-1}$ at

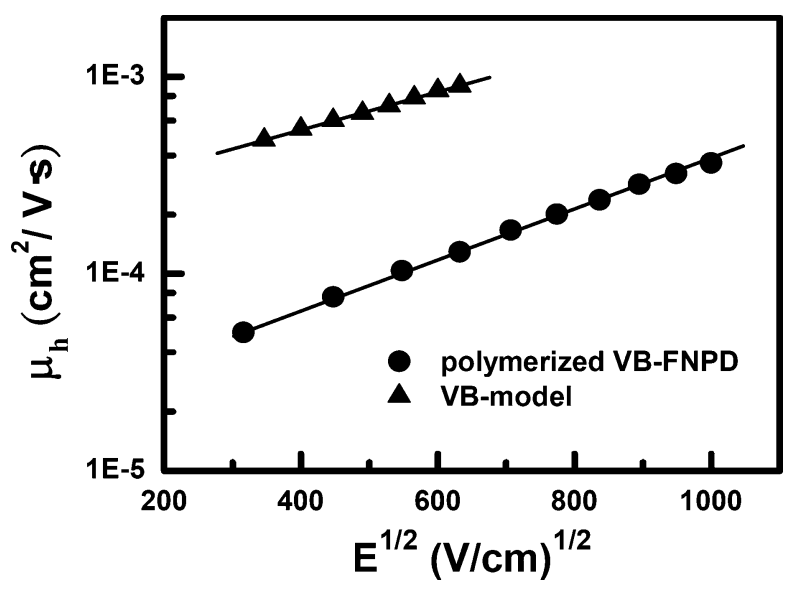

Fig. 5 Hole mobilities within thin films of the VB-FNPD polymer and VB-model plotted as a function of the square root of the electric field.
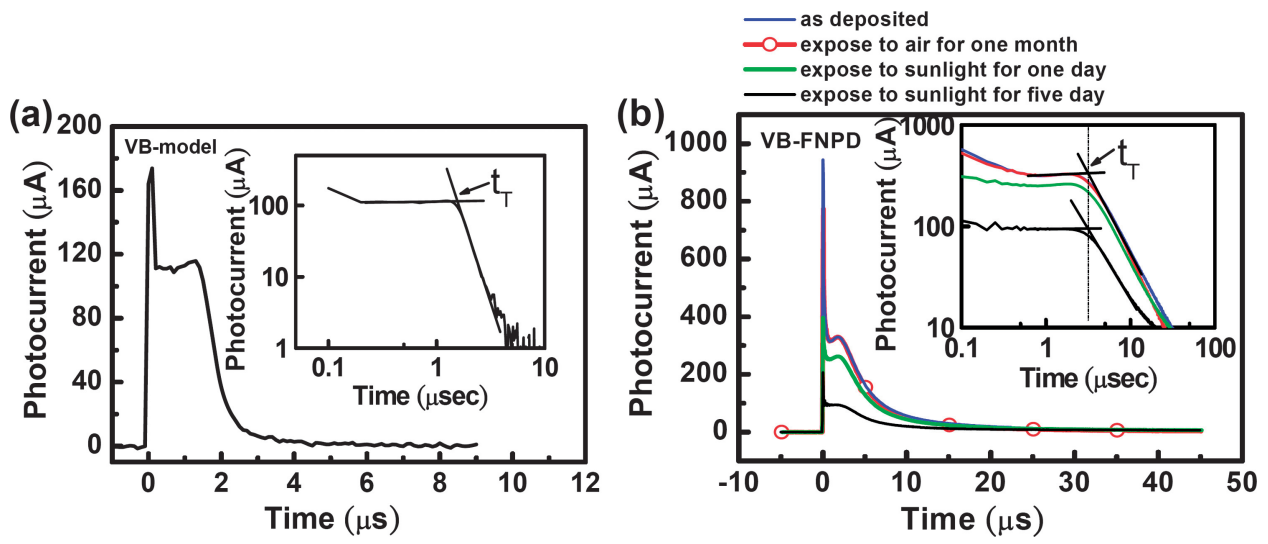

Fig. 4 Non-dispersive transient photocurrents plotted as a function of time of (a) VB-model (thickness: $2.5 \mu \mathrm{m} ; E=2.4 \times 10^{5} \mathrm{~cm} \mathrm{~V}^{-1}$ ) and (b) the polymerized VB-FNPD (thickness: $1 \mu \mathrm{m} ; E=3 \times 10^{5} \mathrm{~cm} \mathrm{~V}^{-1}$ ). Inset: double-logarithmic plots for determination of the transit times. 

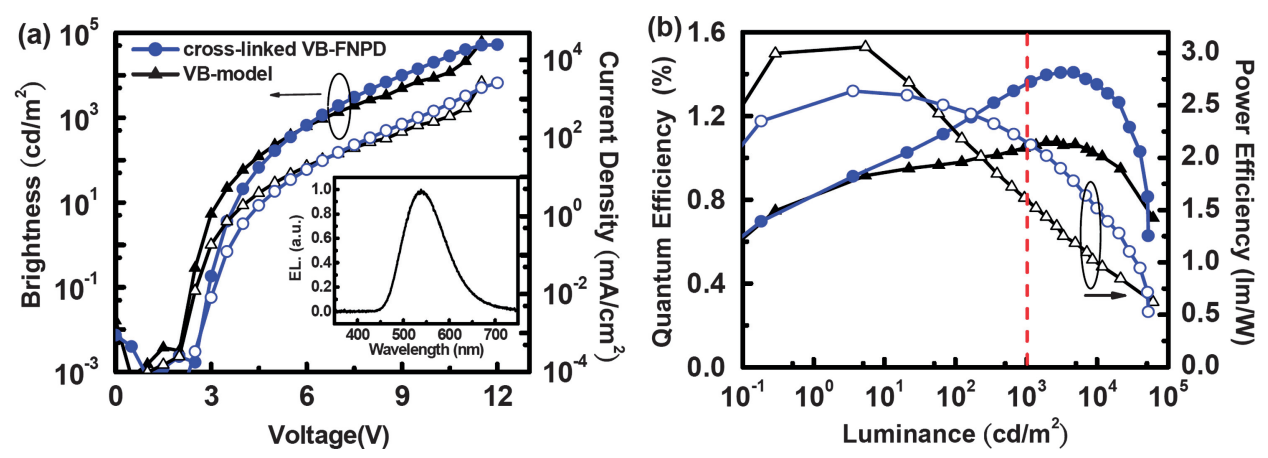

Fig. 6 (a) $I-V-L$ characteristics (inset: EL spectrum) and (b) external quantum and power efficiencies plotted with respect to the luminance for Alq3 devices incorporating the polymerized VB-FNPD and the small molecule VB-model as HTLs.

electric fields between $10^{5}$ and $10^{6} \mathrm{~V} \mathrm{~cm}^{-1}$. The hole mobilities of the VB-FNPD polymer films were about one order of magnitude lower than those of its model counterpart. We attribute this decrease in hole mobility to the polystyrene units within the VBFNPD polymeric films hindering the hopping of the charge carriers among adjacent molecules.

\section{Device}

To assess the feasibility of using VB-FNPD polymeric thin films as hole transporting layers (HTLs), we fabricated hybrid OLEDs having the device structure ITO/PEDOT:PSS/VB-FNPD (50 $\mathrm{nm}) / \mathrm{Alq} 3(50 \mathrm{~nm}) / \mathrm{LiF}(0.5 \mathrm{~nm}) / \mathrm{Al}(100 \mathrm{~nm})$, in which ITO/ PEDOT:PSS and LiF/Al acted as the anode and cathode, respectively, and vacuum-deposited conventional tris(8-quinolinato)aluminium (Alq3) acted as the electron transport/emissive layer. For comparison, the vacuum-deposited VB-model was also used as an HTL in its corresponding device architecture.

Fig. 6 displays the current-voltage-luminance $(I-V-L)$ characteristics, as well as plots of device efficiency versus luminance, for the devices incorporating the polymerized VB-FNPD and VB-model. Both devices exhibit a voltage-independent pure emission from Alq3, implying that charge recombination occurred exclusively within the Alq3 layer [see inset of Fig. 6(a)]. The hybrid OLED incorporating the polymerized VB-FNPD exhibited a turn-on voltage of $2.5 \mathrm{~V}$, a maximum brightness of $52600 \mathrm{~cd} \mathrm{~m}^{-2}$ at $2600 \mathrm{~mA} \mathrm{~cm}^{-2}(12 \mathrm{~V})$, an external quantum efficiency (EQE) of up to $1.4 \%\left(4.5 \mathrm{~cd} \mathrm{~A}^{-1}\right)$ at $100 \mathrm{~mA} \mathrm{~cm}^{-2}$, and a power efficiency of $2.6 \mathrm{~lm} \mathrm{~W}^{-1}$. The device incorporating vacuum-deposited VB-model exhibited a turn-on voltage of $2 \mathrm{~V}$, a similar brightness of $61000 \mathrm{~cd} \mathrm{~m}^{-2}$ at $2690 \mathrm{~mA} \mathrm{~cm}^{-2}(11.5 \mathrm{~V})$, a lower EQE of $1.1 \%\left(3.4 \mathrm{~cd} \mathrm{~A}^{-1}\right)$ at $70 \mathrm{~mA} \mathrm{~cm} \mathrm{~cm}^{-2}$, and a power efficiency of $3 \mathrm{~lm} \mathrm{~W}^{-1}$. Notably, the device based on the polymerized VB-FNPD exhibited superior performance relative to that based on VB-model (Fig. 6b); yet, at the practical brightness of $1000 \mathrm{~cd} \mathrm{~m}^{-2}$, the EQE and power efficiency of the device using polymerized VB-FNPD remain above $1.35 \%$ and $2.11 \mathrm{~mW}^{-1}$, respectively, which are better than those of the device using VBmodel. We attribute the improved performance to the morebalanced charge flux and better exciton confinement within the emission layer that resulted from the presence of the VB-FNPD polymeric thin film as the hole transport layer. Thus, a more balanced recombination of holes and electrons can be achieved with a high EQE of $1.4 \%$, even at a high driving current.

\section{Conclusion}

We have synthesized a fluorene-based triaryldiamine bearing thermally polymerizable pendent styryl groups. The solutionprocessed monomer underwent smooth polymerization upon heating at $170^{\circ} \mathrm{C}$ to give a polymer film displaying photophysical and electrochemical properties similar to those observed prior to thermal treatment. TOF techniques revealed the existence of non-dispersive hole transport in the VB-FNPD polymer film, with the hole mobility reaching as high as $10^{-4} \mathrm{~cm}^{2} \mathrm{~V}^{-1} \mathrm{~s}^{-1}$. This article is the first to report the intrinsic charge carrier mobility of a styrene-based thermally polymerizable material. This new VBFNPD polymer film exhibits remarkable ambient stability and has potential for application as a hole-transporting material in OLEDs. The device fabricated using the solution-processed, thermally cured VB-FNPD polymer film exhibited a maximum EQE $\left(\eta_{\mathrm{ex}}\right)$ of $1.4 \%$, considerably better than that of the device incorporating the vacuum-deposited model compound VBmodel $\left(\eta_{\mathrm{ex}}=1.1 \%\right)$. The high air stability of the VB-FNPD polymer film suggests that it may function as an active material within robust organic optoelectronic devices.

\section{Experimental}

\section{Synthesis}

2: Tri-tert-butylphosphine ( $6 \mathrm{~mL}, 0.05 \mathrm{M}$ in toluene, $0.3 \mathrm{mmol})$ was added to a solution of the dibromide 1 (5.08 g, $10 \mathrm{mmol}), \mathrm{N}$ phenyl-1-naphthylamine $(4.39 \mathrm{~g}, 20 \mathrm{mmol}), \mathrm{Pd}(\mathrm{OAc})_{2}(67 \mathrm{mg}$, $0.3 \mathrm{mmol}$ ), and sodium tert-butoxide $(7.68 \mathrm{~g}, 80 \mathrm{mmol})$ in toluene $(100 \mathrm{~mL})$. The mixture was heated under reflux under Ar for $72 \mathrm{~h}$ and then quenched with water. The solution was partitioned between ethyl acetate $(300 \mathrm{~mL})$ and brine $(100 \mathrm{~mL})$. The combined organic extracts were dried $\left(\mathrm{MgSO}_{4}\right)$ and concentrated. The resulting solid was washed with hexane to afford a yellow product $(6.41 \mathrm{~g}, 82 \%)$. M.p. $254-255^{\circ} \mathrm{C}$. IR $(\mathrm{KBr}) \nu$ 3544 (w), 3055 (w), 1609 (m), 1592 (s), 1573 (m), 1507 (s), 1489 (s), 1464 (s), 1434 (m), 1392 (m), 1302 (m), 1269 (s), 1172 (m), $1015(\mathrm{~m}) \mathrm{cm}^{-1} .{ }^{1} \mathrm{H}$ NMR (DMSO- $\left.d_{6}, 400 \mathrm{MHz}\right): \delta 9.29(\mathrm{~s}, 2 \mathrm{H})$, $7.97(\mathrm{~d}, J=8.0 \mathrm{~Hz}, 2 \mathrm{H}), 7.84(\mathrm{~d}, J=8.4 \mathrm{~Hz}, 2 \mathrm{H}), 7.76(\mathrm{~d}, J=8.4$ $\mathrm{Hz}, 2 \mathrm{H}), 7.56-7.48(\mathrm{~m}, 6 \mathrm{H}), 7.37(\mathrm{t}, J=7.4 \mathrm{~Hz}, 2 \mathrm{H}), 7.28(\mathrm{~d}, J=$ $7.2 \mathrm{~Hz}, 2 \mathrm{H}), 7.16(\mathrm{t}, J=7.6 \mathrm{~Hz}, 4 \mathrm{H}), 6.92-6.84(\mathrm{~m}, 8 \mathrm{H}), 6.78(\mathrm{~s}$, $2 \mathrm{H}), 6.51(\mathrm{~d}, J=8.8 \mathrm{~Hz}, 4 \mathrm{H}), 6.41(\mathrm{~d}, J=8.4 \mathrm{~Hz}, 4 \mathrm{H}) .{ }^{13} \mathrm{C} \mathrm{NMR}$ (DMSO- $\left.d_{6}, 100 \mathrm{MHz}\right): \delta 155.5,152.2,147.5,146.5,142.6,135.3$, 
$134.7,132.9,130.1,129.1,128.4,128.2,126.6,126.4,126.3,126.1$, 123.4, 121.6, 121.1, 120.4, 120.2, 118.8, 114.6, 63.0. MS (FAB ${ }^{+}$, $m / z) 785$ (100), 784 (65), 154 (70), 136 (65), 57 (65). HRMS $\left(\mathrm{FAB}^{+},[\mathrm{M}+\mathrm{H}]^{+}\right)$Calcd. $\mathrm{C}_{57} \mathrm{H}_{41} \mathrm{~N}_{2} \mathrm{O}_{2}$ 785.3170, found 785.3165.

VB-FNPD: 18-Crown-6 (105 mg, $0.4 \mathrm{mmol})$, potassium carbonate (553 mg, $4 \mathrm{mmol}$ ), and 4-vinylbenzyl chloride (610 mg, $4 \mathrm{mmol}$ ) were added to a solution of the bisphenol 2 (785 mg, 1 $\mathrm{mmol})$ in acetone $(16 \mathrm{~mL})$. After heating under reflux for $18 \mathrm{~h}$, the suspension was cooled to room temperature. The salts were filtered off and washed extensively with $\mathrm{CH}_{2} \mathrm{Cl}_{2}(300 \mathrm{~mL})$. After evaporation of the solvent, the residue was dissolved in $\mathrm{CH}_{2} \mathrm{Cl}_{2}$ $(300 \mathrm{~mL})$ and then extracted with brine $(100 \mathrm{~mL} \times 2)$. The combined organic extracts were dried $\left(\mathrm{MgSO}_{4}\right)$ and concentrated. The resulting residue was washed with hexane and methanol. The crude product was purified through column chromatography $\left(\mathrm{SiO}_{2}, \mathrm{CH}_{2} \mathrm{Cl}_{2}\right.$-hexane $\left.2: 3\right)$ to afford the product $(550 \mathrm{mg}, 54 \%)$ as a yellow solid. IR (KBr) $\nu 2955(\mathrm{w})$, 2922 (m), 2851 (w), 1660 (m), 1631 (m), 1592 (m), 1504 (m), 1465 (m), $1391(\mathrm{~m}), 1271(\mathrm{~m}), 1239(\mathrm{w}), 1177(\mathrm{w}), 1106(\mathrm{w}), 826(\mathrm{w})$, $773(\mathrm{w}) \mathrm{cm}^{-1} .{ }^{1} \mathrm{H}$ NMR (DSMO- $d_{6}, 400 \mathrm{MHz}$ ): $\delta 7.95$ (d, $J=8.4$ $\mathrm{Hz}, 2 \mathrm{H}), 7.83(\mathrm{~d}, J=8.4 \mathrm{~Hz}, 2 \mathrm{H}), 7.73(\mathrm{~d}, J=8.4 \mathrm{~Hz}, 2 \mathrm{H}), 7.57$ $(\mathrm{d}, J=8.4 \mathrm{~Hz}, 2 \mathrm{H}), 7.52-7.47(\mathrm{~m}, 8 \mathrm{H}), 7.40-7.34(\mathrm{~m}, 6 \mathrm{H}), 7.27$ $(\mathrm{d}, J=7.2 \mathrm{~Hz}, 2 \mathrm{H}), 7.15(\mathrm{t}, J=7.8 \mathrm{~Hz}, 4 \mathrm{H}), 6.92-6.84(\mathrm{~m}, 8 \mathrm{H})$, $6.80(\mathrm{~s}, 2 \mathrm{H}), 6.79-6.68(\mathrm{~m}, 6 \mathrm{H}), 6.63(\mathrm{~d}, J=8.8 \mathrm{~Hz}, 4 \mathrm{H}), 5.86(\mathrm{~d}$, $J=17.6 \mathrm{~Hz}, 2 \mathrm{H}), 5.27(\mathrm{~d}, J=10.8 \mathrm{~Hz}, 2 \mathrm{H}), 4.99(\mathrm{~s}, 4 \mathrm{H}) \cdot{ }^{13} \mathrm{C}$ NMR (acetone- $\left.d_{6}, 100 \mathrm{MHz}\right): \delta 158.0,153.2,148.9,148.0,144.1$, $138.5,137.7,137.2,136.1,134.4,131.4,129.7,129.5,129.2,128.4$, $127.4,127.1,127.0,126.9,126.8,124.7,122.5,122.4,121.8,120.9$, 120.2, 114.9, 114.2, 70.0, 64.5. MS ( $\left.m / z, \mathrm{FAB}^{+}\right) 1015.7$ (15), 289.0 (10), 154.0 (100), 135.9 (90). HRMS $\left(\mathrm{M}^{+}, \mathrm{FAB}^{+}\right)$Calcd. $\mathrm{C}_{75} \mathrm{H}_{56} \mathrm{~N}_{2} \mathrm{O}_{2}$ 1016.4342, found 1016.4370. Anal. Calcd for $\mathrm{C}_{75} \mathrm{H}_{56} \mathrm{~N}_{2} \mathrm{O}_{2} \mathrm{C}, 88.55 ; \mathrm{H}, 5.55 ; \mathrm{N}, 2.75$; found $\mathrm{C}$, 88.71; H, 5.48; N, 2.34 .

\section{Cyclic voltammetry}

The oxidation potentials were determined by cyclic voltammetry (CV) in $\mathrm{CH}_{2} \mathrm{Cl}_{2}$ solution $(1.0 \mathrm{mM})$ containing $0.1 \mathrm{M}$ tetra- $n$ butylammonium hexafluorophosphate $\left(\mathrm{TBAPF}_{6}\right)$ as a supporting electrolyte at a scan rate of $100 \mathrm{mV} \mathrm{s}^{-1}$. A glassy carbon electrode and a platinum wire were used as the working and counter electrodes, respectively. The ferrocene/ferrocenium redox couples occur at values of $E_{\mathrm{o}}{ }^{\prime}$ of $+0.46 \mathrm{~V}$ in $\mathrm{CH}_{2} \mathrm{Cl}_{2}-$ $\mathrm{TBAPF}_{6}$. All potentials were recorded versus $\mathrm{Ag} / \mathrm{AgCl}$ (saturated) as a reference electrode.

\section{Photophysical property measurements}

Steady state spectroscopic measurements were conducted in solid films. For VB-model, a thin film was prepared by vacuum $(2 \times$ $10^{-6}$ Torr) deposition on a quartz plate $(1.6 \times 1.0 \mathrm{~cm})$. For VBFNPD, a thin film was prepared by dip-coating from a $1-2 \mathrm{wt} \%$ solution in THF, which was subsequently baked at $100{ }^{\circ} \mathrm{C}$ for 30 min, and then heated at $170{ }^{\circ} \mathrm{C}$ for $30 \mathrm{~min}$ to expedite polymerization in a glove box. Absorption spectra were recorded with a U2800A spectrophotometer (Hitachi) and fluorescence spectra were acquired on a F4500 fluorescence spectrophotometer (Hitachi) upon exciting at the absorption maxima.

\section{Ultraviolet photoemission spectroscopy (UPS) measurements}

The valence-band ultraviolet photoemission spectra were carried out with $\mathrm{He}$ I $(21.2 \mathrm{eV})$ and $\mathrm{He}$ II $(40.8 \mathrm{eV})$ as excitation sources. The Fermi level of the system was measured on the gold substrate before the organic deposition. The energy levels of the HOMOs of organic samples were determined by extrapolating the edges of the HOMO peak down to the background of the UPS spectra. The vacuum levels of the films were deduced from the position of the spectra cutoff at the lowest binding energy and the photon energy of excitation sources.

\section{Time-of-flight (TOF) mobility measurements}

TOF samples were prepared by dissolving appropriate weight ratios (up to $25 \mathrm{wt} \%$ ) of the monomer VB-FNPD in THF and then dip-coating the solutions onto an ITO substrate within a glove box. The film thicknesses were controlled by varying the solution concentrations and the dip-coating conditions; the films were subsequently dried through baking at $100^{\circ} \mathrm{C}$ for $30 \mathrm{~min}$ to remove the residual solvent and then cured at $170{ }^{\circ} \mathrm{C}$ for $30 \mathrm{~min}$ to form the polymers. A Dektak surface profilometer was used to measure the thicknesses of the VB-FNPD $(c a .1 \mu \mathrm{m})$ polymer films. The device samples were then completed through thermal deposition of the back electrode $[\mathrm{Ag}(100 \mathrm{~nm})]$ through a shadow mask.

For the TOF measurements, the samples were mounted in a cryostat under vacuum ( $c a \cdot 10^{-3}$ Torr); the temperature was controlled using a PID thermocontroller. A sheet of charge carriers in the organic layer was generated by radiating a short excitation pulse (nitrogen laser) through the semi-transparent ITO electrode. Under an applied DC voltage, these charge carriers sweep over the organic sample toward the counter electrode $(\mathrm{Ag})$ and discharge to result in a transient photocurrent. When the carriers reached the counter electrode, the current dropped to zero; the time at which this event occurred corresponded to the transit time of the carriers. The photocurrent signal was detected using a digital storage oscilloscope. Selected carriers (holes or electrons) drifted across the sample upon switching the polarity of the applied bias. The carrier mobility $(\mu)$ was calculated from the transit time $\left(t_{\mathrm{T}}\right)$, the sample thickness $(d)$, and the applied voltage $(V)$ using the expression $\mu=d^{2} / t_{\mathrm{T}} V$.

\section{Device fabrication}

ITO substrates were cleaned in an ultrasonic detergent bath and then washed sequentially in acetone and methanol. The substrates were subsequently treated in a UV-ozone cleaner to remove any residual organic contaminants. A holeinjection layer of poly(3,4-ethylenedioxythiophene)-poly(4stylenesulfonate) (PEDOT:PSS) was first spin-coated onto the ITO substrate to a thickness of $30 \mathrm{~nm}$ and then dried at $120^{\circ} \mathrm{C}$ for $30 \mathrm{~min}$ to remove residual solvent. After direct dip-coating of a solution of the monomer VB-FNPD (1.2 $\mathrm{wt} \%$ in THF) onto the PEDOT:PSS/ITO substrate, the system was baked at $100{ }^{\circ} \mathrm{C}$ for $30 \mathrm{~min}$ to remove residual solvent and then it was heated at 170 ${ }^{\circ} \mathrm{C}$ for $30 \mathrm{~min}$ to expedite polymerization. A $50 \mathrm{~nm}$ thick layer of Alq3 was then vacuum-deposited on top of the polymerized VBFNPD at a deposition rate of $2-3 \AA \mathrm{s}^{-1}$. Finally, a $0.5 \mathrm{~nm}$ thick layer of $\mathrm{LiF}$ and a $100 \mathrm{~nm}$ thick $\mathrm{Al}$ cathode were deposited through a shadow mask (area: $0.10 \mathrm{~cm}^{2}$ ). OLED device 
characterization was performed at $25{ }^{\circ} \mathrm{C}$ under a nitrogen atmosphere using a computer-controlled Keithley 6430 source meter and a Keithley 6487 picoammeter equipped with a calibrated silicon photodetector. EL spectra were measured using a photodiode array detector (Ocean Optics S2000) over the spectral range of 200 to $850 \mathrm{~nm}$ at a resolution of $2 \mathrm{~nm}$.

\section{References}

1 C. W. Tang and S. A. VanSlyke, Appl. Phys. Lett., 1987, 51, 913.

2 D. W. Steuerman, A. Garcia, M. Dante, R. Yang, J. P. Löfvander and T.-Q. Nguyen, Adv. Mater., 2008, 20, 528.

3 H. B. Wu, F. Huang, Y. Q. Mo, W. Yang, D. L. Wang, J. B. Peng and Y. Cao, Adv. Mater., 2004, 16, 1826; W. L. Ma, P. K. Iyer, X. Gong, B. Liu, D. Mose, G. C. Bazan and A. J. Heeger, Adv. Mater., 2005, 17, 274; X. Gong, S. Wang, D. Moses, G. C. Bazan and A. J. Heeger, $A d v$. Mater., 2005, 17, 2053.

4 U. Schubert, N. Hüsing and A. Lorenz, Chem. Mater., 1995, 7, 2010; W. Li, Q. Wang, J. Cui, H. Chou, S. E. Shaheen, G. E. Jabbour, J. Anderson, P. Lee, B. Kippelen, N. Peyghambarian, N. R. Armstrong and T. J. Marks, Adv. Mater., 1999, 11, 730; Q. Huang, G. A. Evmenenko, P. Dutta, P. Lee, N. R. Armstrong and T. J. Marks, J. Am. Chem. Soc., 2005, 127, 10227; J. G. C. Veinot and T. J. Marks, Acc. Chem. Res., 2005, 38, 632; J. Li and T. J. Marks, Chem. Mater., 2008, 20, 4873.

5 C. D. Müller, A. Falcou, N. Reckefuss, M. Rojahn, V. Wiederhirn, P. Rudati, H. Frohne, O. Nuyken, H. Becker and K. Meerholz, Nature, 2003, 421, 829; E. Bacher, M. Bayerl, P. Rudati, N. Reckefuss, C. D. Müller, K. Meerholz and O. Nuyken, Macromolecules, 2005, 38, 1640; M. C. Gather, A. Köhnen, A. Falcou, H. Becker and K. Meerholz, Adv. Funct. Mater., 2007, 17, 191; P. Zacharias, M. C. Gather, M. Rojahn, O. Nuyken and K. Meerholz, Angew. Chem., Int. Ed., 2007, 46, 4388; B. Domercq, R. D. Hreha, Y.-D. Zhang, N. Larribeau, J. N. Haddock,
C. Schultz, S. R. Marder and B. Kippelen, Chem. Mater., 2003, 15, 1491.

6 M.-Y. Chou, M.-K. Leung, Y. O. Su, C. L. Chiang, C.-C. Lin, J.-H. Liu, C.-K. Kuo and C.-Y. Mou, Chem. Mater., 2004, 16, 654; C.-C. Chang and M.-K. Leung, Chem. Mater., 2008, 20, 5816; C. Xia and R. C. Advincula, Chem. Mater., 2001, 13, 1682; S. Inaoka, D. B. Roitman and R. C. Advincula, Chem. Mater., 2005, 17, 6781.

7 S. Liu, X. Jiang, H. Ma, M. S. Liu and A. K.-Y. Jen, Macromolecules, 2000, 33, 3514; X. Jiang, S. Liu, M. S. Liu, P. Herguth, A. K.-Y. Jen, H. Fong and M. Sarikaya, Adv. Funct. Mater., 2002, 12, 745; Y. Niu, M. S. Liu, J.-W. Ka and A. K.-Y. Jen, Appl. Phys. Lett., 2006, 88, 093505; J. Zhao, J. A. Bardecker, A. M. Munro, M. S. Liu, Y. Niu, I.-K. Ding, J. Luo, B. Chen, A. K.-Y. Jen and D. S. Ginger, Nano Lett., 2006, 6, 462; B. Lim, J.-T. Hwang, J. Y. Kim, J. Ghim, D. Vak, Y.-Y. Noh, S.-H. Lee, K. Lee, A. J. Heeger and D.-Y. Kim, Org. Lett., 2006, 8, 4703; G. K. Paul, J. Mwaura, A. A. Argun, P. Taranekar and J. R. Reynolds, Macromolecules, 2006, 39, 7789.

8 Y.-H. Niu, M. S. Liu, J.-W. Ka, J. Bardeker, M. T. Zin, R. Schofiled, Y. Chi and A. K.-Y. Jen, Adv. Mater., 2007, 19, 300; Y.-H. Niu, A. M. Munro, Y.-J. Cheng, Y. Tian, J. Zhao, J. A. Bardecker, J.-L. Plante, D. S. Ginger and A. K.-Y. Jen, Adv. Mater., 2007, 19, 3371; Y.-J. Cheng, M. S. Liu, Y. Zhang, Y.-H. Niu, F. Huang, J.-W. Ka, H.-L. Yip and A. K.-Y. Jen, Chem. Mater., 2008, 20, 413.

9 F. Huang, Y.-J. Cheng, Y. Zhang, M. S. Liu and A. K.-Y. Jen, J. Mater. Chem., 2008, 18, 4495.

10 Y.-L. Liao, W.-Y. Hung, T.-H. Hou, C.-Y. Lin and K.-T. Wong, Chem. Mater., 2007, 19, 6350.

11 Y. Shirota and H. Kageyama, Chem. Rev., 2007, 107, 953.

12 K.-T. Wong, Z.-J. Wang, Y.-Y. Chien and C.-L. Wang, Org. Lett., 2001, 3, 2285.

13 C.-H. Chou and C.-F. Shu, Macromolecules, 2002, 35, 9673.

14 P. M. Borsenberger and D. S. Weiss, Organic Photoreceptor for Imaging Systems, Marcel Dekker, New York, 1993. 